\title{
COATINGS FOR THE APS RF CAVITY TUNERS FOR THE REDUCTION OF SECONDARY ELECTRONS *
}

\author{
G. Harris, Q. Ma, D. R. Walters ${ }^{\#}$ \\ Argonne National Laboratory, Advanced Photon Source \\ 9700 South Cass Avenue, Argonne, Illinois 60439 USA
}

\begin{abstract}
Thin film coatings are used to reduce the generation of secondary electrons at the ends of the Advanced Photon Source (APS) rf cavity tuners because these electrons result in increased temperatures on the tuner. To improve the film properties over the existing evaporated titanium film, a magnetron-sputtered film of titanium nitride has been developed that is both harder and adheres more strongly than the past evaporated film. This paper describes the process for creating this new coating.
\end{abstract}

\section{INTRODUCTION}

The Advanced Photon Source (APS) is a facility for material science research. It provides users with a source of X-rays at an energy of $7 \mathrm{GeV}$. The main positron beam is contained within the storage ring that is twothirds of a mile in circumference. The storage ring is divided into 40 sectors with isolation valves between each sector. Sixteen $\mathrm{rf}$ cavities are used to maintain the electron beam energy in the APS storage ring at $7 \mathrm{GeV}$. In each cavity there is a tuner piston, as seen in Fig. 1, that can be extended or retracted to match the cavity's resonance to the required frequency.

\section{RF TUNERS}

The rf cavity tuner is a copper piston that, when moved, changes the volume of the cavity; this in turn changes the resonance of the cavity. From the electrical field point of view this method of tuning is lossy, and with the high field gradients, many aspects of the design need to be optimized to keep the tuner temperatures under control [1]. The problem of electron heating of the tuner in the annulus between it and the tuner sleeve can be severe if the surfaces of the piston are not correctly prepared. In the annulus, electrons coming from the high electrical field of the cavity strike the copper of the piston and produce secondary electrons. These in turn bounce around and again strike the piston, producing more secondary electrons. Every strike of electrons heats the tuner more, so if the surface can be modified not to produce secondary electrons, the temperature of the tuner will be reduced. This paper reports on a thin film coating

* Work supported by the U.S. Department of Energy, Office of Basic Energy Sciences, under Contract No. W-31-109-ENG-38.

\# Email: drw@ aps.anl.gov that reduces the secondary electrons produced on the surface of the copper piston and aids in the control of the tuner temperature.

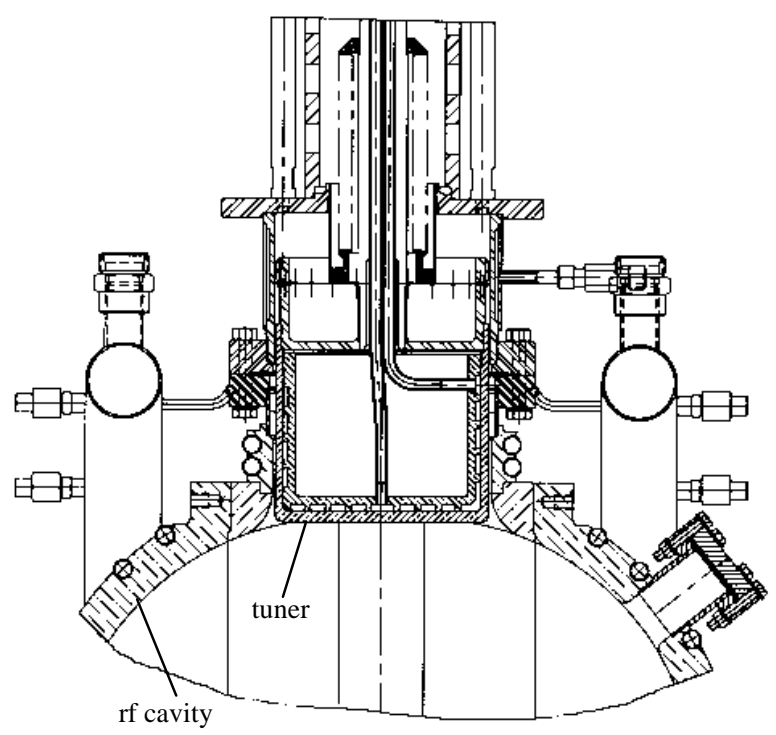

Figure 1: Rf cavity with tuner

\section{TITANIUM NITRIDE COATING}

\subsection{Coating System Description}

The coating system used at the APS is pictured in Fig. 2. It consists of a main bell jar, into which the tuner is loaded, on top of a base plate where the pump and the sputtering gun are also mounted. The tuner is placed on a turntable so that it can be rotated in front of the sputtering gun while the thin film coating is being applied. The turntable has the added characteristic of being electrically isolated from the system. The turntable and tuner can then have a biasing voltage applied to it to alter the properties of the thin film. This became important while developing of the coating process because it directly altered the surface of the tuner during the coating process.

The magnetron sputtering gun used in this system is a 1.5 inch $\times 8$ inch linear type gun that is mounted 6 inches from the tuner. The power for the gun comes from a 2.5$\mathrm{kW}$ DC power supply connected to an Advanced Energy Sparc-le ${ }^{\mathrm{TM}}$ unit. The Sparc-le ${ }^{\mathrm{TM}}$ unit adds a $20-\mathrm{kHz}$ AC signal to the main power that helps in the coating of 


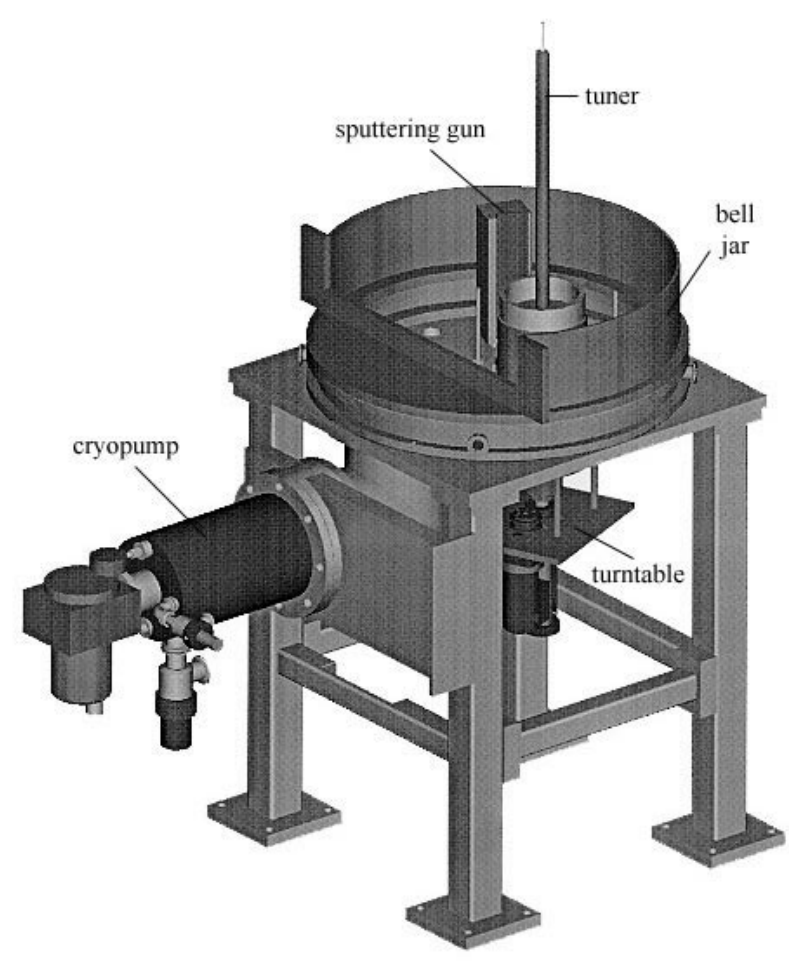

Figure 2: Coating system

insulating films. To apply voltage to the tuner, a $5-\mathrm{kW}$ DC power supply is configured so that the voltage range of the bias supply was adjustable between 0 and 1,000 volts.

The gas inlet system is made up of two MKS Model 1179A mass flow controllers: one for argon, 200 standard cubic centimeters per minute $(\mathrm{sccm})$, and one for nitrogen, $20 \mathrm{sccm}$. On each line there is a high efficiency gas filter as well as a gas purifier to remove gaseous contaminates. The total system pressure is measured using a capacitance diaphragm gauge. A 2-Torr head was chosen so that readings in the milliTorr range would be repeatable and reliable.

The vacuum system is comprised of a $9 \mathrm{cfm}$ dry pump for roughing and a cryopump as the main high vacuum pump. The cryopump was throttled by a VAT Series 64 valve to obtain the desired process pressure independently of gas flow into the system.

\subsection{Titanium Nitride Process}

Reactive sputtering is a process where the nitrogen and titanium are brought to the tuner surface and a stoichiometric compound of titanium nitride forms on it [2]. The sputtering gun is the source of titanium for the coating. However, the nitrogen, $\mathrm{N}_{2}$, is a nearly inert gas and must be changed to atomic nitrogen in order to form titanium nitride.

In most cases the sputtering gun's glow discharge is energetic enough to break the $\mathrm{N}_{2}$ bonds to produce $\mathrm{N}^{+}$ ions. This did not work in our case because the distance the ions had to travel before striking the surface of the tuner was sufficient for the nitrogen to reform into $\mathrm{N}_{2}$. At the process pressure of 20 milliTorr the resulting mean free path is $2.5 \mathrm{~mm}$. With a distance between the gun and tuner of 60 mean-free-path lengths, it is highly unlikely that the atomic nitrogen will get from the gun to the tuner surface. Over time, what was found to work was a secondary glow discharge on the tuner that would locally dissociate nitrogen so that titanium nitride could form on the tuner surface. Applying a high voltage on the tuner made the glow, which cracked the nitrogen. The energy of the glow is high enough that the nitrogen's bonds are broken right over the surface that is being coated. In this way the atomic nitrogen only needs to travel a few millimeters before it hits the tuner surface.

The completeness of the nitrogen dissociation was judged by the color of the film. A bright gold color was considered an indication that the film composition was approximately correct. Other means were also employed to more fully qualify the film. The surface science technique of using Auger signatures was used to make the final determination of the quality of the film composition. A series of tests confirmed that the best film was produced with the tuner at 700 VDC.

The other set-up parameters for the film are: gun wattage of 400 watts, bias voltage of $700 \mathrm{VAC}$, argon flow rate of $50 \mathrm{sccm}$, system pressure of 20 milliTorr, and a nitrogen gas flow rate of $0.8 \mathrm{sccm}$.

\subsection{Characteristics of Thin Film}

The new film being developed needed to be an improvement over the evaporated film that had been made in the past. The evaporated film suffered from poor adhesion, brittleness, low strength, and was easily scratched. The goal of the new film was to use a method that would solve the adhesion problem and increase the film strength and hardness. Those last two characteristics could be solved by controlling the composition of the coating to a higher degree than was done in the past.

The film that resulted from the set-up described in the previous section greatly improves upon the earlier film. The process of sputtering while biasing the tuner immediately improved the adhesion. The final film process produces a film that adheres so well it cannot be pulled off with scotch tape and is so hard that a knife with a steel blade can hardly scratch it.

To verify the composition of the coating, a comparison was performed between a known sample of titanium nitride and a sample from this machine. There is a very high correlation between the two samples, as seen in Fig. 3. The upper peaks show the known sample and the lower peaks are from our process using $0.8 \mathrm{sccm}$ of $\mathrm{N}_{2}$. The coating itself was gold in color with a shiny finish. We will be testing its secondary electron yield in the future, but reports [3] concerning its performance show that the $\sigma$, yield, is less than 0.4 for TiN and $\sigma \cong 1.0$ for titanium. This is an improvement over copper, which has a $\sigma$ of 1.3 [4]. 


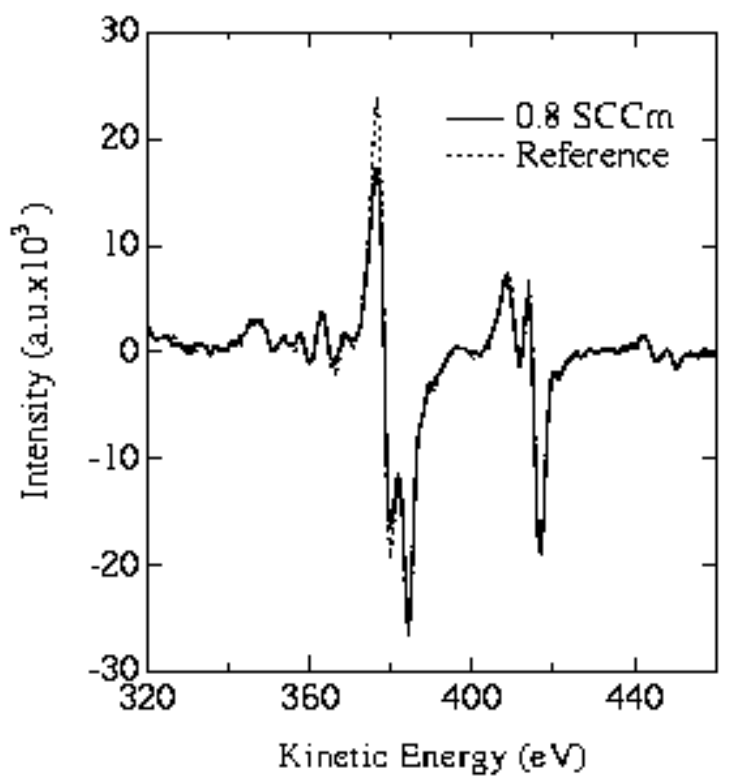

Figure 3: Auger comparison of reference and 0.8 sccm TiN film

\section{CONCLUSIONS}

The coating produced by the process described here is a great improvement over the old process. The new coating is very robust and does not suffer from handling damage as the old one did. With the improved adherence there is no flaking of the film as there once was. Overall, the new process provides a better film that, when installed into the rf cavity, behaves closer to its theoretical capabilities.

\section{ACKNOWLEDGMENTS}

The authors would like to thank Cathy Eyberger for her help in compiling and editing this paper. We have had the guidance of Joseph Gagliano and John Noonan (ANL) in the details of the design of the system. The process development was greatly aided by suggestions from Mark Bernick of Angstrom Sciences.

\section{REFERENCES}

[1] J. Jones, D. Bromberek, Y. Kang, R. Kustom, L. Morrison, R. Piech, E. Rotela, S. Sharma, I.C. Sheng, Proceedings of the 1997 Particle Accelerator Conference, Vancouver, B.C., pp. 29-29 (1997).

[2] W.D. Sproul, P.J. Rudnik, and M.E. Graham, "The Effect of $\mathrm{N}_{2}$ Partial Pressure, Deposition Rate and Substrate Bias Potential on the Hardness and Texture of Reactively Sputtered TiN Coatings," Surface and Coatings Technology, 39/40, p. 355 (1989).

[3] M. A. Lewis, D. A. Glocker, J. Jorne, "Measurement of Secondary Electron Emission in Reactive Sputtering of Aluminum and Titanium Nitride," J. Vac. Sci. Technol. A 7(3), p. 1019, May/Jun 1989.

[4] CRC Handbook of Chemistry and Physics, 64 ${ }^{\text {th }}$ Edition. Ed Robert Weast. Boca Raton: CRC Press, 1983. 